\title{
Comprehensive Geriatric Assessment among demented and non-demented patients with chronic kidney disease
}

Ola Abdelfattal Amr1, El-Banouby M. H1, Ibrahim M.2, Walaa W. Aly1, Suzan Mounir Ali1.

1 Geriatrics and Gerontology Department, Faculty of Medicine, Ain Shams University, Cairo, Egypt.

2 Internal Medicine and Nephrology Department, Faculty of Medicine, Ain Shams University, Cairo, Egypt

\begin{abstract}
Background: Chronic kidney disease (CKD) prevalence is increasing among elderly populations. Elderly patients with CKD are more likely to have multiple comorbidities, and more liable to complications and increased mortality. Cognitive impairment is common among patients with CKD; however, the impact of this association on the burden of diverse geriatric conditions e.g. depression, malnutrition, and functional dependency is unclear. Being a multidimensional, interdisciplinary diagnostic process, Comprehensive Geriatric Assessment (CGA) can capture the full spectrum of comorbidities in CKD patients with cognitive decline.
\end{abstract}

Objective: to detect the prevalence of geriatric impairments using the Comprehensive Geriatric Assessment among demented and non-demented patients with CKD.

Methods: A cross-sectional study conducted on 30 elderly patients with CKD, not on haemodialysis. Cognitive functions assessment was done using Mini Mental State examination (MMSE) and Montreal Cognitive assessment (MoCA), then the patients were reclassified as demented and non-demented according to MMSE and MoCA. Other comprehensive geriatric assessment domains (depression, function and nutritional status) were compared between both groups. Basic laboratory investigations were obtained.

Results: Cognitive functions assessment of CKD patients by MMSE showed that $56.67 \%$ ( $n=17$ ) were normal and $43.33 \%(n=13)$ showed cognitive impairment and by using MoCA revealed that $63.33 \%(n=19)$ were normal, while $36.67 \%(n=11)$ were cognitively impaired. No statistically significant difference between CKD demented and nondemented patients classified according to (MMSE) and MoCA as regard demographic data. Statistically significant difference was found between CKD demented and non-demented patients classified according to MMSE and MoCA as regard Activities of Daily Livings (ADLs), Instrumental Activities of Daily Livings (IADLs), Mini Nutritional Assessment (MNA) and depression assessed by GDS15. No statistically significant difference was found between CKD demented and non-demented patients classified according to MMSE and MoCA as regard laboratory investigations as Hemoglobin, urea, Creatinine, $\mathrm{Na}$, k, Total calcium, phosphorus, intact PTH, Albumin.

Conclusion: Demented CKD patients had more depression, functional decline, malnutrition in comparison to nondemented patients.

Keywords: Cognitive functions, Chronic Kidney disease, Elderly

\section{Background}

The prevalence of chronic kidney diseases (CKD) is increasing among elderly populations. Data from the National Health and Nutrition Examination Survey (NHANES) suggest that chronic kidney disease (CKD) prevalence is highest in the elderly; the prevalence is $38 \%$ in participants aged > 65 years compared with $13 \%$ in the overall US population [1]. Cognitive functions impairment has been recognized as a complication of end stage renal disease (ESRD)[2]
The accumulation of uremic toxins may cause cerebral endothelial dysfunction and contribute to cognitive disorders in CKD [3].

Various uremic toxins have been implicated in the pathogenesis of cognitive impairment [4]. It has been reported that cerebrospinal fluid and brain levels of some guanidine compounds, such as creatinine, guanidine, guanidine-succinic acid, and methylguanidine, are substantially elevated in uremic patients 
[5]. Interestingly, these high toxin concentrations (up to 10 -fold higher in CKD patients than in controls) were found in brain regions that play a determinant role in cognition, such as the thalamus, the mammillary bodies, and the cerebral cortex [5]. It is well known that these uremic guanidine compounds are neuro excitatory agents with convulsing activity.

However, it is still not clear whether uremic toxins are directly responsible for cognitive impairment. The involvement of guanidine in cognitive disorders could also be indirect because it has been shown that these compounds favor an elevation of serum homocysteine [6].

It has been showed that community-resident elderly individuals with elevated levels of Cystatin-C (an inhibitor of cysteine proteases that co-localizes with $\beta$ amyloid in the brain of patients with Alzheimer disease) had lower cognitive test scores and were more likely to experience a decline in cognitive function. It is possible that Cystatin- $\mathrm{C}$ has a direct effect on the risk of developing Alzheimer disease [7].

The accelerated cognitive decline in older CKD patients appears to be due to the CKD disease process itself, which creates a toxic vascular and metabolic milieu that consists of chronic inflammation, oxidative stress, uremia, and systemic vascular endothelial dysfunction [8]. This toxic internal vascular and metabolic milieu is postulated to cause vascular dysfunction related impairment of the white matter that is superimposed on neurodegenerative damage caused by homocysteine, uremic toxins, creatinine, and cystatin C [9].

There is an association between baseline albuminuria and incident cognitive impairment at higher estimated glomerular filtration rate (eGFR) levels, while among individuals with minimal albuminuria but with lower eGFR, the eGFR itself was more predictive of cognitive risk [10]. Albuminuria was associated with both worse executive functioning and greater white matter hyper intensity burden on brain imaging [11]. An evaluation of the Cardiovascular Health Study cohort, showed a $22 \%$ increased risk of dementia with each doubling of the urine albumin-creatinine ratio [12].

A Comprehensive Geriatric Assessment is a systematic procedure that is designed to assess the health of the elderly population by focusing on somatic, functional, social and psychosomatic domains. It reveals deficits that are not routinely captured in standard history and examination and has been proven to be valuable for improving survival and functional outcomes in different categories of elderly patients [13].

All patients being evaluated for cognitive impairment or dementia should be screened for depression. Cognitive impairment may be the chief complaint in a patient with depression. Depression can also worsen cognitive impairment in patients with dementia or mild cognitive impairment (MCI). It is not uncommon for a depressed person to literally elicit a depressed feeling in the clinician examining that patient. Several screening tools have been validated e.g. the Patient Health Questionnaire 2 (PHQ2), which consists of just two questions [14].

Cognitive impairment impacts patients negatively by contributing to functional dependence and behavioral symptoms that result in poor outcomes and decreased medication and medical care compliance. These negative consequences result in a downward spiral of functional decline and an accelerated loss of independence, which leads to premature institutionalization [15]. The negative impact of cognitive impairment on quality of life and emotional wellbeing is significant, and it even affects employment rates negatively [16]. Moreover, cognitive impairment more than doubles mortality risk and increases days spent in the hospital [17], contributing to the tremendous individual, societal, and economical burden of CKD, that is why the aim of this study is to assess the prevalence of geriatric impairments among demented and non-demented patients with chronic kidney diseases.

\section{Methods}

\section{Study population}

A cross sectional study was conducted on 30 elderly patients with CKD not on haemodialysis aged 60 years and older attending the Ain Shams University Hospital, Cairo, Egypt. The subjects who refused or could not complete the interview were excluded from the study. Those with disturbed conscious level or delirium and those with decompensated chronic liver disease (ChildPugh Classification Stage B and C), and with major visual or hearing impairment were excluded from the study.

\section{Ethical consideration:}

The study methodology was reviewed and approved by the Research Review Board of the Geriatrics and Gerontology Department, Faculty of Medicine, Ain Shams University. Informed consent was taken from every subject participating in this study.

\section{Clinical assessment:}

All participants were subjected to comprehensive geriatric assessment. Detailed socio-demographic data, medical history, surgical history and physical examination were done. Blood pressure and body mass index (BMI) were measured.

\section{- Cognitive functions assessment}

Cognitive functions assessment was done by using the Arabic version by El-Okl et al [18] of Mini-mental status examination (MMSE) [19] and Arabic version by Rahman TT and El Gaafary MM [20] of Montreal Cognitive Assessment (MoCA) [21] and accordingly the patients were reclassified as demented and nondemented according to MMSE and MoCA,

The MMSE comprises 30 items which examine 
orientation (10 points), registration of new information ( 3 points), attention and calculation ( 5 points), recall ( 3 points), assessing language skills (8 points) and construction question (1 point). MMSE score was then adjusted to age and education.

Scoring: Mini-mental state examination score on a scale was $0-30$ and more than 25 was interpreted as normal cognitive functions, mild cognitive impairment from 18 to 23 and severe cognitive impairment from 0 to 17 .

MoCA is thirty points tool which administered in 10 minutes. MoCA examine different cognitive domains.

The short-term memory recall domain (5 points), Visuospatial functions were assessed using a clock drawing test ( 3 points) and a three-dimensional cube figure (1 point). Trail-making B test (1 point), two item verbal abstraction (2 points) and a verbal fluency (1 point). Working memory and attention are examined by administration of a sustained attention duty ( 1 point), a serial subtraction (3 points), and digit span forward and backward (1 point each). Assessment of language was done by a three-item naming duty with unfamiliar animals ( 3 points), repetition of two syntactically complex sentences ( 2 points), and the previously mentioned fluency mission. Finally, orientation to time and place is assessed (6 points).

Scoring: The cut off MoCA in CKD was 23.5.

- Assessment of other Geriatric Domains

We compare the two groups (demented and nondemented CKD patients) as regard the other geriatric domains to detect the prevalence of geriatric impairments among the 2 groups.

a) All participants were screened for depression using the Arabic version by Shehata et al., [22] of geriatric depression scale (GDS) [23]. GDS-15 was done to exclude any depressive symptoms. The cut-off point is (5) for significant depressive symptoms.

b) Functional assessment was done using activities of daily livings (ADLs) [24] and instrumental IADLs scales [25].ADLs assess the patient abilities to complete basic self-care tasks. The results of this assessment were as follows: The lowest score is 0 and indicates dependence while the highest score is 6 and indicates independence. Instrumental activities of daily livings (IADLs) scale are abilities of patient to keep independent. The results of this assessment tool were as follows: The score ranges from 0 (low function, dependence) to 8 (high function, independence).

c) Nutritional Assessment was done using the Arabic version by AbdElaziz et al., [26] of Mini Nutritional Assessment (MNA) [27]. Full form of MNA administer in 2 step: screening then assessment. Screening by MNA-short form and provide six item checklist dealing with appetite, weight loss, mobility, illness, Neuro-psychological problems and body mass index. MNA-short form maximum score was 14 . If screening score on MNA-SF was 12 points or more, elderly classify as well-nourished and no need for assessment complete. Otherwise if cases are 11 points or less, cases require to complete assessment part (MNA). Scoring: Full MNA maximum score is 30 . Elderly receive more 24 indicating that elderly was well-nourished, Between 17 and 23.5 indicated a risk of malnutrition and a score less than 17 points score indicated malnutrition.

\section{- Laboratory Investigations}

Laboratory investigations including haemoglobin level, urea, creatinine, sodium, potassium, total calcium, phosphorus, intact parathyroid hormone (PTH) and albumin. Fasting serum samples were obtained.

\section{Statistical Analysis}

Data entry and statistical analysis by SPSS statistical software package (SPSS Inc., Chicago, IL). Chi square test to compare the difference between 2 qualitative variables. Independent sample t-test to compare the significance of differences for parametric variable between 2 independent means. Paired samples t-tests for statistical significance assessment of sample of matched pairs of similar units or one group of units which tested twice.

\section{Results}

Mean age of the participants was $66.367 \pm 4.760$ years. Male patients were $53.33 \%(n=16)$ and female patients were $46.67 \%(n=14)$.

Cognitive functions assessment by MMSE showed that $56.67 \%(n=17)$ of the subjects were normal and $43.33 \%$ $(\mathrm{n}=13)$ were demented. By using MoCA, $63.33 \%$ $(n=19)$ of subjects were normal, while $36.67 \%(n=11)$ were demented. No statistically significant difference between demented and non-demented patients (classified according to Mini Mental State Examination) as regard demographic data (Table 1). Statistically significant difference was found between CKD non-demented and demented patients classified according to (MMSE) as regard Activities of Daily Livings (ADLs) as $53.86 \%$ of demented CKD patients are assisted in ADL versus $0 \%$ of non-demented CKD patients, also statistically significant difference was found between CKD non-demented and demented patients as regard Instrumental Activities of Daily Livings (IADLs) as $53.85 \%$ of demented CKD patients are assisted in IADL versus $11.76 \%$ of non-demented CKD patients, also statistically significant difference was found between CKD non-demented and demented patients as regard Mini Nutritional Assessment (MNA) as $38.46 \%$ of demented CKD patients are malnourished versus $11.76 \%$ non-demented CKD patients, Statistically significant difference was found between CKD non-demented and demented patients as regard GDS 15 (Table 2). 
Table 1: Comparison of the CKD patients divided according to Mini Mental State Examination (MMSE) as regard demographic data

\begin{tabular}{|c|c|c|c|c|c|c|c|c|c|}
\hline \multirow{3}{*}{\multicolumn{2}{|c|}{ CKD patients }} & \multicolumn{6}{|c|}{ MMSE score } & \multicolumn{2}{|c|}{ Chi-Square } \\
\hline & & \multicolumn{2}{|c|}{ Normal } & \multicolumn{2}{|c|}{ Dementia } & \multicolumn{2}{|c|}{ Total } & \multirow[b]{2}{*}{$\mathbf{X}^{2}$} & \multirow[b]{2}{*}{ P-value } \\
\hline & & $\mathbf{N}$ & $\%$ & $\mathbf{N}$ & $\%$ & $\mathbf{N}$ & $\%$ & & \\
\hline \multirow[t]{2}{*}{ Gender } & Male & 11 & 64.71 & 5 & 38.46 & 16 & 53.33 & \multirow[t]{2}{*}{2.039} & \multirow[t]{2}{*}{0.153} \\
\hline & Female & 6 & 35.29 & 8 & 61.54 & 14 & 46.67 & & \\
\hline \multirow[t]{5}{*}{ Smoking } & Yes & 8 & 47.06 & 5 & 38.46 & 13 & 43.33 & \multirow[t]{2}{*}{0.222} & \multirow[t]{2}{*}{0.638} \\
\hline & No & 9 & 52.94 & 8 & 61.54 & 17 & 56.67 & & \\
\hline & \multirow[t]{3}{*}{ CKD patients } & \multicolumn{6}{|c|}{ MMSE score } & \multirow{2}{*}{\multicolumn{2}{|c|}{ T-Test }} \\
\hline & & \multicolumn{3}{|c|}{ Normal } & \multicolumn{3}{|c|}{ Dementia } & & \\
\hline & & Mean & \pm & SD & Mean & \pm & SD & $\mathbf{t}$ & P-value \\
\hline & Age (years) & 65.000 & \pm & 3.953 & 68.154 & \pm & 5.273 & -1.875 & 0.071 \\
\hline & BMI (kg/m2) & 26.524 & \pm & 3.302 & 23.654 & \pm & 3.831 & 2.201 & 0.076 \\
\hline & Systolic BP (mmHg) & 131.765 & \pm & 11.311 & 132.308 & \pm & 9.268 & -0.141 & 0.889 \\
\hline & Diastolic BP (mmHg) & 80.000 & \pm & 6.124 & 78.462 & \pm & 3.755 & 0.797 & 0.432 \\
\hline
\end{tabular}

CKD: Chronic Kidney Diseases, BP; blood pressure, BMI: body mass index

Table 2: Comparison of the CKD patients divided according to Mini Mental State Examination (MMSE) as regard Comprehensive Geriatric Assessment

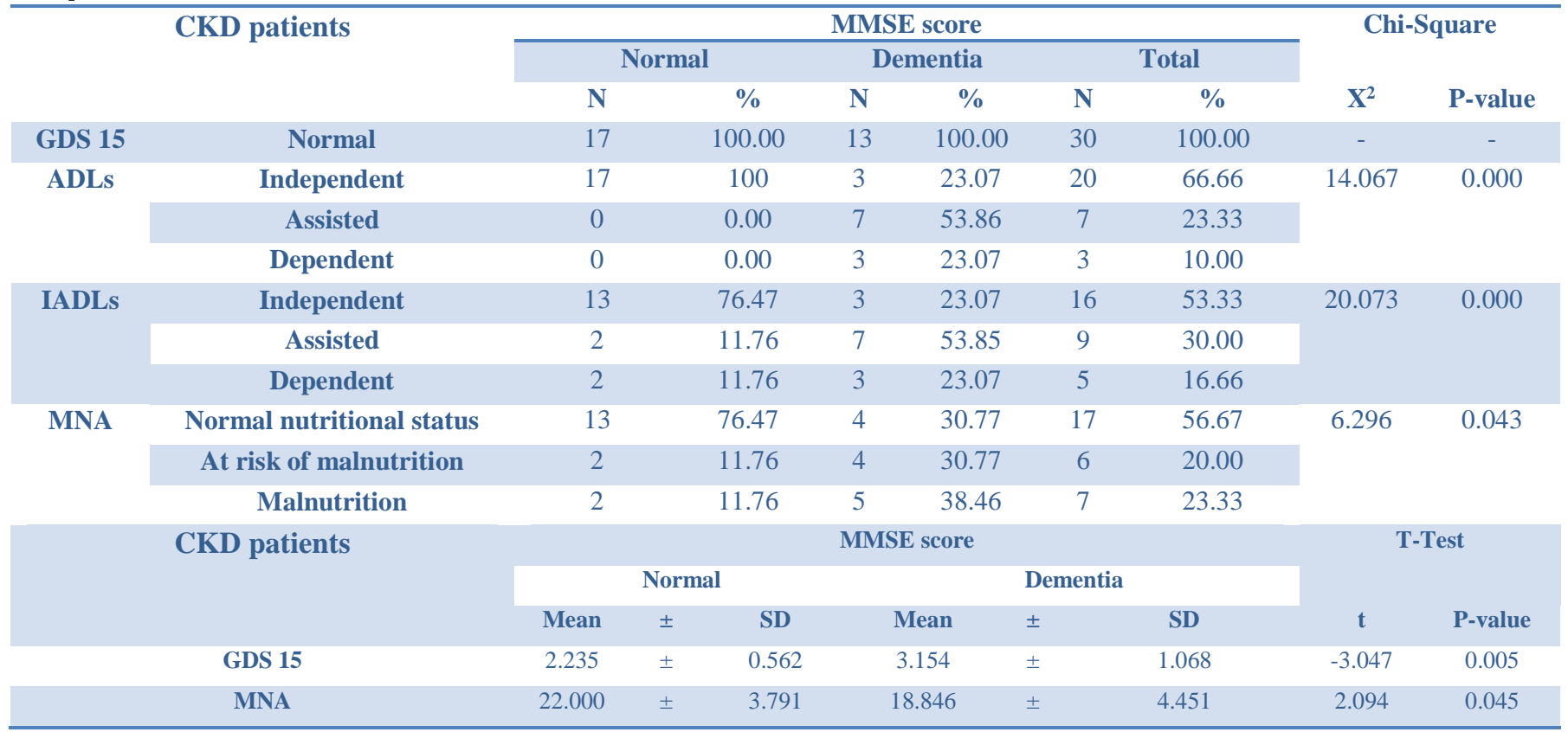

CKD: Chronic Kidney Diseases, ADLs; Activities of Daily Livings, IADLs: Instrumental Activities of Daily Livings, MNA: MiniNutritional Assessment, GDS15; Geriatric Depression Scale 15. 
Table 3: Comparison of the CKD patients divided according to Mini Mental State Examination (MMSE) as regard laboratory investigations

\begin{tabular}{|c|c|c|c|c|c|c|c|c|c|c|}
\hline \multirow[t]{3}{*}{ CKD patients } & \multicolumn{7}{|c|}{ MMSE score } & \multirow{2}{*}{\multicolumn{3}{|c|}{ T-Test }} \\
\hline & \multicolumn{3}{|c|}{ Normal } & \multicolumn{4}{|c|}{ Dementia } & & & \\
\hline & Mean & \pm & \multicolumn{2}{|l|}{ SD } & Mean & \pm & SD & $\mathbf{T}$ & \multicolumn{2}{|r|}{ P-value } \\
\hline HGB (g/dl) & 10.447 & \pm & \multicolumn{2}{|l|}{0.883} & 10.354 & \pm & 0.631 & 0.322 & \multicolumn{2}{|r|}{0.749} \\
\hline Urea (mg/dl) & 80.765 & \pm & \multicolumn{2}{|l|}{45.464} & 109.308 & \pm & 45.481 & -1.704 & \multicolumn{2}{|r|}{0.100} \\
\hline BUN (mg/dl) & 37.691 & \pm & \multicolumn{2}{|l|}{21.218} & 51.010 & \pm & 21.227 & -1.703 & \multicolumn{2}{|r|}{0.100} \\
\hline $\mathrm{Cr}$ (mg/dl) & 1.612 & \pm & \multicolumn{2}{|l|}{0.451} & 2.223 & \pm & 0.611 & -3.156 & \multicolumn{2}{|r|}{0.295} \\
\hline $\mathrm{Na}(\mathrm{mmol} / \mathrm{dl})$ & 135.118 & \pm & 1.269 & & 135.385 & \pm & 1.850 & -0.469 & & 0.643 \\
\hline $\mathrm{K}(\mathrm{mmol} / \mathrm{dl})$ & 4.329 & \pm & 0.761 & & 4.523 & \pm & 0.784 & -0.682 & & 0.501 \\
\hline T.Ca (mg/dl) & 8.294 & \pm & 0.651 & & 7.892 & \pm & 0.830 & 1.487 & & 0.148 \\
\hline Corrected Ca (mg/dl) & 8.335 & \pm & 0.784 & & 8.000 & \pm & 0.943 & 1.064 & & 0.297 \\
\hline Po4 (mg/dl) & 7.047 & \pm & 2.084 & & 6.908 & \pm & 2.884 & 0.154 & & 0.879 \\
\hline CaPo4 Product & 58.368 & \pm & 17.355 & & 54.762 & \pm & 22.002 & 0.502 & & 0.619 \\
\hline Intact PTH (pg/dl) & 90.412 & \pm & 56.672 & & 106.615 & \pm & 45.613 & -0.842 & & 0.407 \\
\hline Alb (g/dl) & 3.935 & \pm & 0.392 & & 3.838 & \pm & 0.388 & 0.673 & & 0.506 \\
\hline CKD & ents & & & & & score & & & & -Square \\
\hline & & & & mal & & ntia & & tal & & \\
\hline & & & $\mathbf{N}$ & $\%$ & $\mathbf{N}$ & $\%$ & $\mathbf{N}$ & $\%$ & $\mathbf{X}^{2}$ & P-value \\
\hline HBsAg & Negative & & 17 & 100.00 & 13 & 100.00 & 30 & 100.00 & - & - \\
\hline HIVAB & Negative & & 17 & 100.00 & 13 & 100.00 & 30 & 100.00 & - & - \\
\hline HCVAB & Negative & & 14 & 82.35 & 11 & 84.62 & 25 & 83.33 & 0.027 & 0.869 \\
\hline & Positive & & 3 & 17.65 & 2 & 15.38 & 5 & 16.67 & & \\
\hline
\end{tabular}

CKD: Chronic Kidney Diseases, HGB; Hemoglobin, BUN; Blood Urea Nitrogen, Cr; Creatinine, Na; Sodium, K; Potassium, T.CA; Total Calcium, Po4; Phosphorus, PTH; Parathyroid Hormone. Alb; Albumin.

Table 4: Comparison of the CKD patients divided according to Montreal Cognitive Assessment (MoCA) as regards demographic data

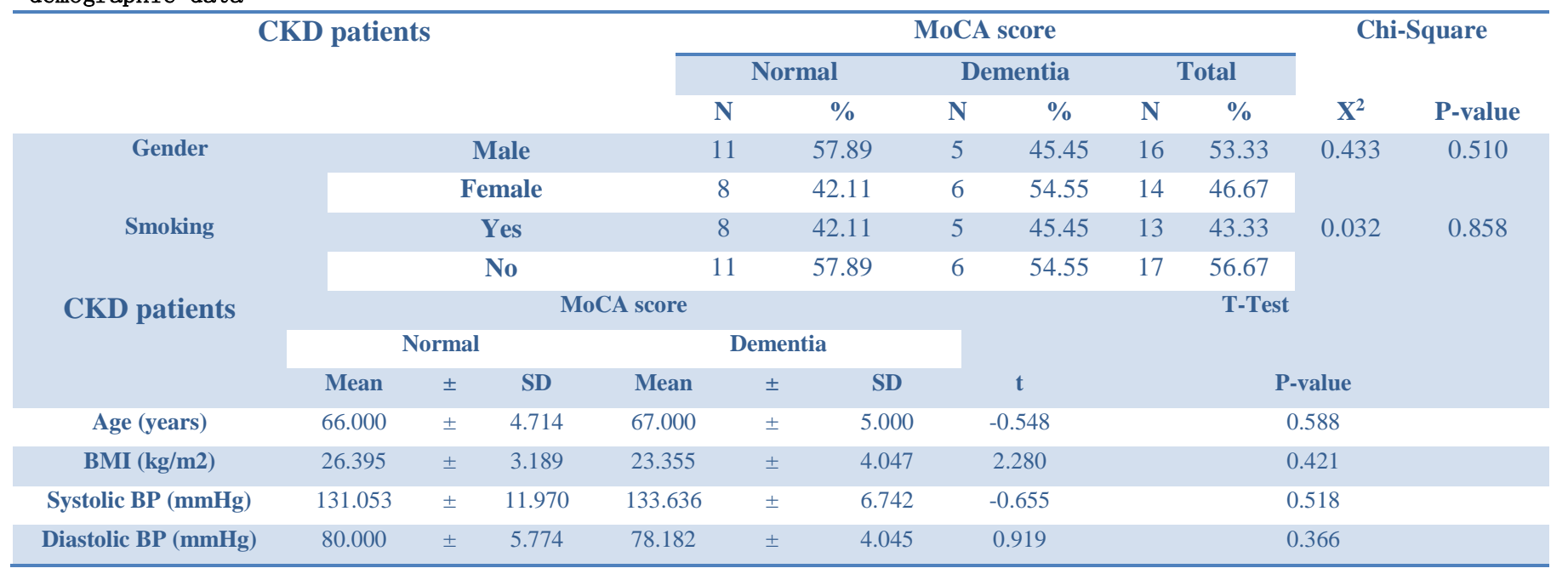

CKD: Chronic Kidney Diseases, BP; blood pressure, BMI: body mass index 
Table 5: Comparison of the CKD patients divided according to Montreal Cognitive Assessment (MoCA) as regard Comprehensive Geriatric Assessment

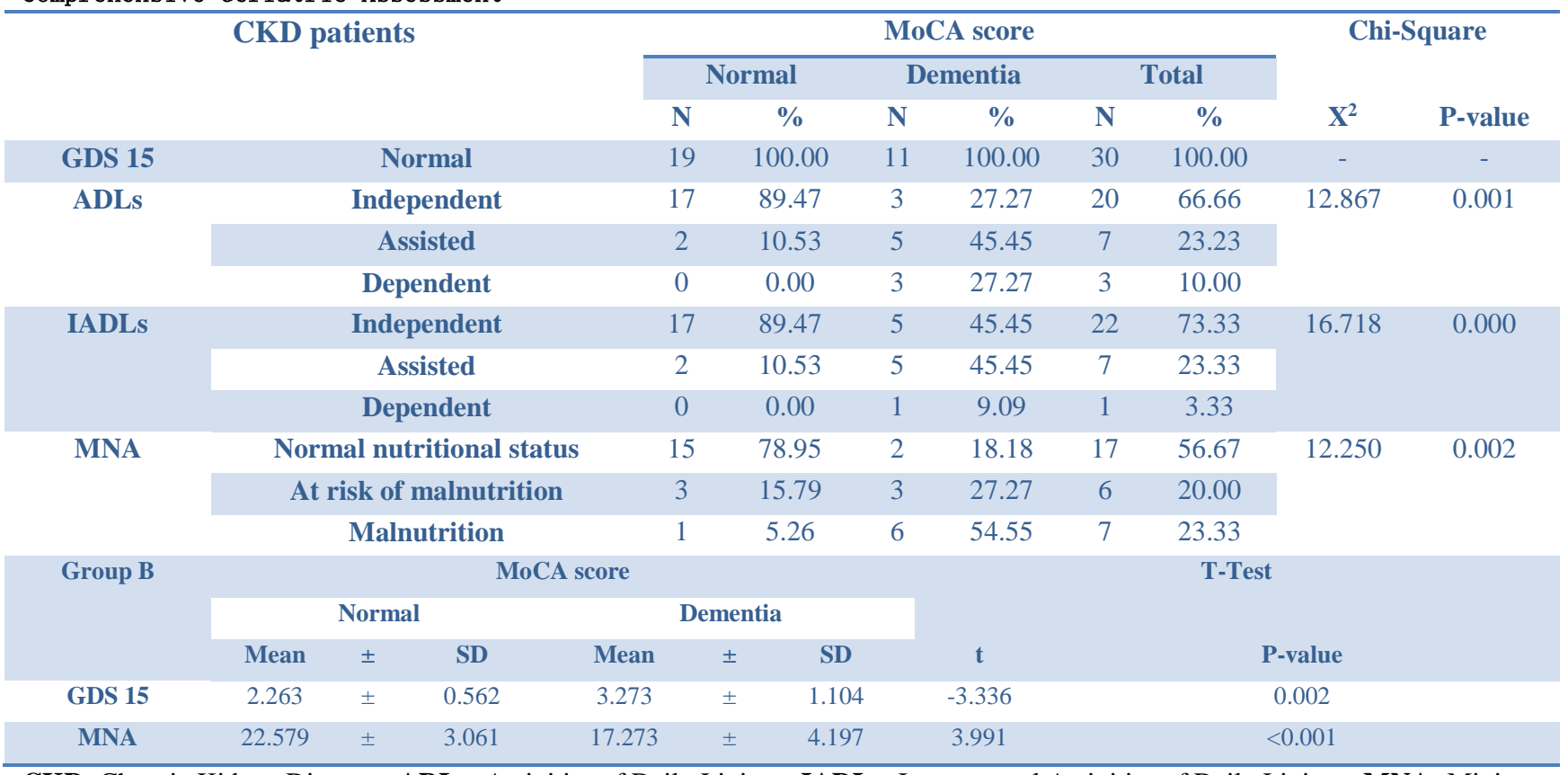

CKD: Chronic Kidney Diseases, ADLs; Activities of Daily Livings, IADLs: Instrumental Activities of Daily Livings, MNA: Mini Nutritional Assessment, GDS15; Geriatric Depression Scale 15.

Table 6: Comparison of the CKD patients divided according to Montreal Cognitive Assessment (MoCA) as regard laboratory investigations

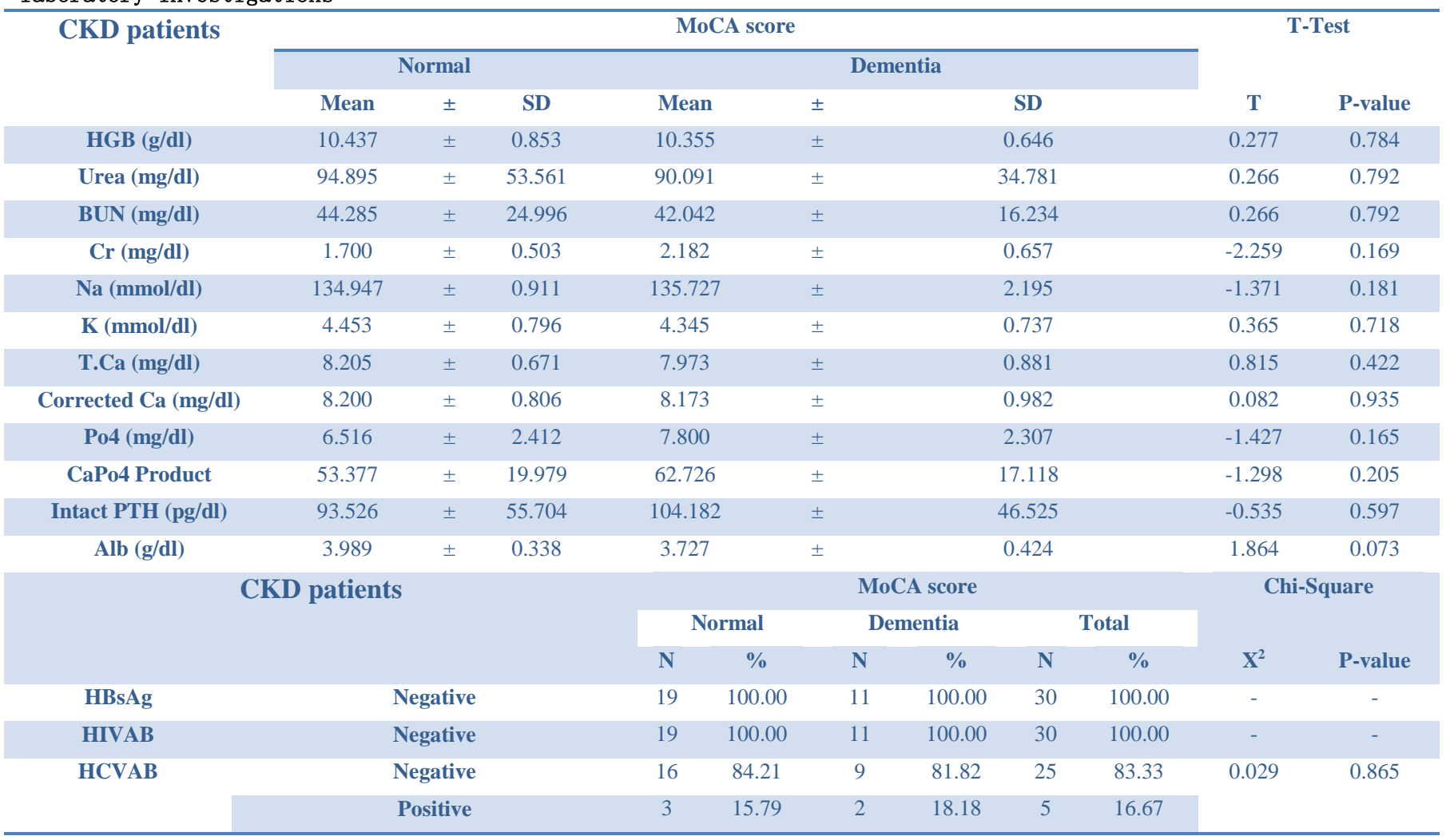

CKD: Chronic Kidney Diseases, HGB; Hemoglobin, BUN; Blood Urea Nitrogen, Cr; Creatinine, Na; Sodium, K; Potassium, T.CA; Total Calcium, Po4; Phosphorus, PTH; Parathyroid Hormone. Alb; Albumin. 
No Statistically significant difference between nondemented and demented patients classified according to (MMSE) regarding HGB, urea, Creatinine, Na, k, Total calcium, phosphorus, intact PTH, Albumin (table 3)

No statistically significant difference between CKD non-demented and demented patients classified according to Montreal Cognitive Assessment (MoCA) as regard demographic data (Table 4). Statistically significant difference was found between CKD nondemented and demented patients classified according to Montreal Cognitive Assessment (MoCA) as regard Activities of Daily Livings (ADLs) as $45.45 \%$ of demented CKD patients are assisted in ADL versus $10.53 \%$ of non-demented CKD patients, also statistically significant difference was found between CKD non-demented and demented patients as regard Instrumental Activities of Daily Livings (ADLs) as $45.45 \%$ of demented CKD patients are assisted in IADL versus $10.53 \%$ of non-demented CKD patients, also statistically significant difference was found between CKD non-demented and demented patients as regard Mini Nutritional Assessment (MNA) as 54.55\% of demented CKD patients are malnourished versus 5.26\% non-demented CKD patients, Statistically significant difference was found between CKD non-demented and demented patients as regard GDS 15 (Table 5).

No Statistically significant difference was found between CKD non-demented and demented patients classified according to (MoCA) as regard laboratory investigations as $\mathrm{HGB}$, urea, Creatinine, Na, k, Total calcium, phosphorus, intact PTH, Albumin (Table 6)

\section{Discussion}

Patients with CKD have increased levels of oxidative stress, caused by uremia, production of reactive oxygen species via physiological pathways (e.g., impaired/ damaged or malfunctioning mitochondria), and an inability to produce adequate anti oxidative enzymes [28]. These changes all contribute to a vascular milieu that consists of systemic inflammation, high levels of oxidative stress, and endothelial dysfunction that is unique to the CKD patient and creates a vascular pathway to cognitive decline [29].

In our study, the overall prevalence of cognitive impairment among CKD patients was $43.33 \%$ by MMSE and $36.67 \%$ by using MoCA and there was statistically significant difference between CKD non-demented and demented patients classified according to (MMSE) and (MoCA) as regard Activities of Daily Livings (ADLs), Instrumental Activities of Daily Livings (IADLs), Mini Nutritional Assessment (MNA) and depression assessed by GDS15

This went in agreement with Carlos et al., who examined Two hundred and fifty-one patients. The average age was $76.3(\mathrm{SD}=7.9)$ years and $86.5 \%$ had CKD stage 3 . Overall prevalence of cognitive impairment (CI) was $51 \%$ (95\% CI 44.7 to 57.2), and the prevalence of major depression reached $8 \%(95 \%$ CI 4.5 to 11.3$) .4 .8 \%$ of the patients $(n=12)$ had both $\mathrm{CI}$ and depression. CI and depression are prevalent conditions among patients with CKD stages 3-4, with the greatest occurrence of CI, affecting half of the investigated Colombian patients with age $\geq 55$ years $[30]$.

This was in consistent with Dalila et al., who evaluated cross-sectional study on 132 elderly subjects (age $\geq$ 65 years) with advanced CKD (stage 4-5). Malnutrition was associated with the severity of cognitive impairment as assessed by MMSE $(\mathrm{B}=-0.19, p<.01)[31]$.

In our study, cognitive functions assessment of CKD patients by MMSE showed that $56.67 \% \quad(n=17)$ were normal and $43.33 \%(n=13)$ showed cognitive impairment and by using MoCA revealed that $63.33 \%(n=19)$ were normal, while $36.67 \%(n=11)$ were cognitively impaired.

This was in agreement with Hailpern and his co-workers who documented that cognitive dysfunction was not limited to hemodialysis patients but it extends to the early stages of kidney disease with a negative correlation between higher brain cognitive functions domains and glomerular filtration rate (GFR) [32].

Besides, Kurella et al., study showed that CKD, with eGFR less than $60 \mathrm{ml} / \mathrm{min}$ per $1.73 \mathrm{~m} 2$, is associated with an increased risk for cognitive impairment [33].

A similar magnitude of increased risk for cognitive impairment was reported for participants with eGFR below $45 \mathrm{ml} / \mathrm{min} / 1.73 \mathrm{~m} 2$ in the Intervention Project on Cerebrovascular Diseases and Dementia in the Community of Ebersberg (INVADE) study. Of the 886 older adults participating in the Rush Memory and Aging project, those with eGFR below $60 \mathrm{ml} / \mathrm{min} / 1.73 \mathrm{~m} 2$ experienced a faster decline in cognitive function, particularly in the memory domains, compared with participants without CKD [34].

Even participants with mild decrement in kidney function experience modestly higher risk. For example, in an analysis from the Health Aging and Body Composition study, participants with eGFR above $60 \mathrm{ml} / \mathrm{min} / 1.73 \mathrm{~m} 2$ but high cystatin $\mathrm{C}$ levels-indicating early CKDexperienced a 1.7-fold higher incidence of cognitive impairment [35].

On the other hand, our study finding disagreed with Helmer et al., who reported that the baseline estimated glomerular filtration rate less than $60 \mathrm{ml} / \mathrm{min}$ per $1.73 \mathrm{~m} 2$, were not associated with an increased risk for incident dementia or cognitive decline [36].

In the same way, we disagreed with Slinin et al., study which failed to find a correlation between cognitive functions impairment and basal glomerular filtration rate at the end of their study [37].

The Rancho Bernardo study among 1,345 adults with an average age of 75 reported that low eGFR was not independently associated with cognitive decline [38].

To the best of our knowledge, Namiko et al is one of the largest studies that used a very extensive Geriatric assessment (GA) to study multiple geriatric domains in patients with ESKD [39]. The prevalence of geriatric impairment in their study is difficult to compare with other similar studies because every study using a GA in this population addressed a different selection of geriatric domains, using various tests and varying cut-off points. 
Despite this, all studies reported a high prevalence of different geriatric impairments [40,41]. However, compared to some of these studies, Namiko et al found a relatively high rate of functional dependency (79\% IADL dependency vs $60 \%$ and $26 \%$ in other studies $[40,42]$

The findings that are obtained by the GA can be used for multiple purposes. First, in studies focusing on one or more geriatric domains in patients with CKD, a reduced functional performance, cognitive impairment, depression were all associated with negative outcomes, such as (early) mortality and hospitalizations [43]. Therefore, the recognition of geriatric impairments could potentially help to direct treatment decisions regarding the start of dialysis and can also help to direct treatment goals with patients and family (e.g., decisions concerning the start of strict fluid restriction, start of new medication and diet) [44]. Second, the identification of geriatric impairments may guide preventive interventions. For instance, when functional impairment, malnutrition or accidental falling are present, it could initiate rehabilitation, nutritional interventions and fall prevention programs, which could potentially improve health outcomes and/or quality of life. Furthermore, early recognition of cognitive impairment allows for diagnosis and appropriate treatment, education, and psychosocial support.

Conclusion: Demented CKD patients had more depression, functional decline, malnutrition in comparison to non-demented patients.

\section{Conflicts of interest}

There were no conflicts of interest regarding publication of our research.

\section{Funding}

The authors received no financial support for the research or authorship of this article.

\section{Availability of data and material}

All the data and material supporting the results of our research will be provided upon request

\section{Ethical Considerations}

This study was accepted by Ain Shams University Ethical Committee. Informed consent was taken from the patient or his caregiver

\section{References}

1. Coresh J, Selvin E, Stevens LA, et al. Prevalence of chronic kidney disease in the United States. JAMA. 2007; 298:20382047.

2. Sarnak MJ, Tighiouart H, Scott TM, et al. Frequency of and risk factors for poor cognitive performance in hemodialysis patients. Neurology. 2013; 80(5): 471-80.

3. Duron $E$, Hanon 0 , et al. Vascular risk factors, cognitive decline, and dementia. Vasc Health Risk Manag. 2008; 4: 363381.

4. Seifter JL, Samuels MA, et al. Uremic encephalopathy and other brain disorders associated with renal failure. Semin Neurol. 2011; 31: 139-143.

5. De Deyn PP, Vanholder R, Eloot S, Glorieux G, et al. Guanidino compounds as uremic (neuro)toxins. Semin Dial. 2009; 22: 340-345.

6. Perna AF, Ingrosso D, Satta E, Lombardi C, Galletti P,
D'Aniello A, De Santo, et al. Plasma protein aspartyl damage is increased in hemodialysis patients: Studies on causes and consequences. J Am Soc Nephrol. 2004; 15: 2747-2754.

7. Kristine Yaffe, Karla Lindquist, Michael G Shlipak, Eleanor Simonsick, et al. Cystatin $\mathrm{C}$ as a Marker of Cognitive Function in Elders: Findings From the Health ABC Study. Ann Neurol. 2008;63(6):798-802.

8. Himmelfarb J: Uremic toxicity, oxidative stress, and hemodialysis as renal replacement therapy. Seminars in Dialysis.2009; 22:636-643.

9. Bugnicourt, JM Godefroy, O Chillon, JM Choukroun, et al. Cognitive disorders and dementia in CKD: the neglected kidney-brain axis. Journal of the American Society of Nephrology.2013; 24:353-363.

10. Kurella Tamura M, Muntner P, Wadley V, et al. Albuminuria, kidney function, and the incidence of cognitive impairment among adults in the United States. Am J Kidney Dis. 2011;58(5):756-763.

11. Weiner DE, Bartolomei K, Scott T, et al. Albuminuria, cognitive functioning, and white matter hyperintensities in homebound elders. Am J Kidney Dis. 2009; 53(3): 438-47.

12. Barzilay JI, Fitzpatrick AL, Luchsinger J, Yasar S, Bernick C, Jenny NS, Kuller LH, et al. Albuminuria and dementia in the elderly: a community study. Am J Kidney Dis. 2008; 52(2):21626.

13. Ellis G, Whitehead MA, Robinson D, O'Neill D, Langhorne P. Comprehensive geriatric assessment for older adults admitted to hospital: meta-analysis of randomised controlled trials. BMJ. 2011;343: d6553.

14. Kroenke K, Spitzer RL, Williams JB, et al. The Patient Health Questionnaire-2: validity of a two-item depression screener. Med Care. 2003; 41:1284.

15. D. E. Weiner and S. L. Seliger, "Cognitive and physical function in chronic kidney disease," Current Opinion in Nephrology and Hypertension, vol. 23, no. 3, pp. 291-297, 2014.

16. L. Feng, K. B. Yap, and T. P. Ng, "Depressive symptoms in older adults with chronic kidney disease: mortality, quality of life outcomes, and correlates," American Journal of Geriatric Psychiatry, vol. 21, no. 6, pp. 570-579, 2013.

17. A. R. Sehgal, S. F. Grey, P. B. DeOreo, and P. J. Whitehouse, "Prevalence, recognition, and implications of mental impairment among hemodialysis patients," American Journal of Kidney Diseases, vol. 30, no. 1, pp. 41-49, 1997.

18. El-Okl MA., Elbanoby MH., Eletrby MA., et al., Prevalence of Alzheimer dementia and other causes of dementia in Egyptian elderly. MD thesis, Faculty of Medicine, Ain Shams University. Geriatric Department Library, 2002.

19. Folstein MF., Folstein SE., and McHugh PR., Mini-mental state. A practical method for grading the cognitive state of patients for the clinician. J Psychiatr Res.1975;12(3):189-98.

20. Rahman TT., EI Gaafary MM. Montreal Cognitive Assessment Arabic version: reliability and validity prevalence of mild cognitive impairment among elderly attending geriatric clubs in Cairo. GeriatrGerontol Int. 2009;9(1):54-61.

21. Nasreddine ZS., Phillips NA., Bédirian V., et al., The Montreal Cognitive Assessment, MoCA: a brief screening tool for mild cognitive impairment. J Am Geriatr Soc. 2005; 53(4): 695-9.

22. Shehata AS., Elbanoby MH., Mortagy AK and Ghanem M., Prevalence of depression among Egyptian Geriatric Community. Ain Shams University. Geriatric Department Library .1998.

23. Sheikh JA and Yesavage YA., Geriatric Depression Scale. Recent evidence anddevelopment of a shorter version. Clinical Gerontology: A Guide to Assessment and Intervention. NY: The Haworth Press.1986; 165-173.

24. Katz S., Ford AB., Moskowitz RW., et al., Studies of illness in the aged. The index of ADL: A standardized measure of biological and psychosocial function. JAMA. 1963; 21(9): 185:914-9.

25. Lawton MP and Brody EM., Assessment of older people: selfmaintaining andinstrumental activities of daily living. Gerontologist. 1969; 9 (3): 179-186.

26. AbdElaziz K. M., AbouHashem R. M., and Abd Al-Atty M. F., Prevalence of malnutrition in recently hospitalized elderly in Cairo using a valid and reliable short form of Arabic version of Mini-Nutritional Assessment (MNA-SF-A). Middle East Journal of Age and Ageing. 2012; 9(4): 8-12.

27. Guigoz, Y., Vellas, B., and Garry, P. J., Assessing the nutritional status of the elderly: The Mini Nutritional 
Assessment as part of the geriatric evaluation. Nutrition reviews. 1996; 54(1): S59.

28. Fujisaki, K Tsuruya, K Yamato, et al. Cerebral oxidative stress induces spatial working memory dysfunction in uremic mice: neuroprotective effect of tempol. Nephrology Dialysis Transplantation. 2014; 29: 529-538.

29. Salisbury $D$ and Bronas U: Reactive oxygen and nitrogen species: impact on endothelial dysfunction. Nursing Research. 2015;64: 53-66.

30. Carlos Edgardo Rodríguez-Angarita, Rafael Mauricio SanabriaArenas, Juan Diego Vargas-Jaramillo, et al. Cognitive impairment and depression in a population of patients with chronic kidney disease in Colombia: a prevalence study. Canadian Journal of Kidney Health and Disease. 2016; 3, 26.

31. Dalila Guenzani, MassimilianoBuoli, LaraCaldiroli, et al. Malnutrition and inflammation are associated with severity of depressive and cognitive symptoms of old patients affected by chronic kidney disease. Journal of Psychosomatic Research.2019;124.

32. Hailpern M., Melamed L., Cohen W and Hostetter H., Moderate chronic kidney disease and cognitive function in adults 20 to 59 years of age: Third National Health and Nutrition Examination Survey (NHANES III). J Am SocNephrol. 2007; 18: 2205-2213.

33. Kurella Tamura M., Wadley V., Yaffe K., et al., Kidney function and cognitive impairment in US adults: The Reasons for Geographic and Racial Differences in Stroke (REGARDS) Study. Am J Kidney Dis.2008; 52: 227-234.

34. Buchman, AS Tanne, D Boyle, PA Shah, RC Leurgans, et al. Kidney function is associated with the rate of cognitive decline in the elderly. Neurology. 2009; 73(12): 920-927.

35. Yaffe K, Lindquist K, Shlipak MG, Simonsick E, Fried L, Rosano $C$, et al. Cystatin $C$ as a marker of cognitive function in elders: Findings from the health $A B C$ study. Ann Neurol. 2008; 63: 798-802.

36. Helmer C, Stengel B, Metzger M, et al. Chronic kidney disease, cognitive decline, and incident dementia: the 3C Study. Neurology. 2011; 77:204351.

37. Slinin Y, Paudel L, Ishani A, et al. Osteoporotic Fractures in Men Study Group: Kidney function and cognitive performance and decline in older men. J Am Geriatr Soc. 2008; 56: 20822088.

38. Jassal, SV Devins, GM Chan, et al. Improvements in cognition in patients converting from thrice weekly hemodialysis to nocturnal hemodialysis: a longitudinal pilot study. Kidney Int.2006;70: 956-962.

39. Namiko A. Goto, Ismay N. van Loon, Moira I. Morpey et al. Geriatric Assessment in Elderly Patients with End-Stage Kidney Disease. Nephron Clin Pract. 2019 Jan; 141(1): 41-48.

40. Parlevliet JL, Buurman BM, Hodac Pannekeet MM, Boeschoten EM, ten Brinke L, Hamaker ME. Systematic comprehensive geriatric assessment in elderly patients on chronic dialysis: a cross-sectional comparative and feasibility study. BMC Nephrol. 2012:13-30.

41. Soysal P, Isik AT, Buyukaydin B, Kazancioglu R. A comparison of end-stage renal disease and Alzheimer's disease in the elderly through a comprehensive geriatric assessment. Int Urol Nephrol. 2014;46:1627-1632.

42. Racic M, Petkovic N, Bogicevic K, Maric I, Matovic J, Pejovic V. Comprehensive geriatric assessment: comparison of elderly hemodialysis patients and primary care patients. Ren Fail. 2015;37:1126-1131.

43. van Loon IN, Wouters TR, Boereboom FT, Bots ML, Verhaar $M C$, Hamaker ME. The relevance of geriatric impairments in patients starting dialysis: a systematic review. Clin J Am Soc Nephrol. 2016;11:1245-1259

44. Jassal SV, Watson D. Dialysis in late life: benefit or burden. Clin J Am Soc Nephrol. 2009;4:2008-2012. 